\title{
Using statistical smoothing to estimate the psycholinguistic acceptability of novel phrases
}

\author{
MATTHEW A. J. ROBERTS \\ University of Edinburgh, Edinburgh, Scotland \\ AND \\ Nick Chater \\ University College London, London, England
}

\begin{abstract}
Native speakers of languages perceive differences in the acceptability of phrases even when those phrases are both grammatical and novel (previously unseen). We suggest that smoothing, a statistical technique used by natural language processing engineers, provides several candidate mechanisms for investigating this phenomenon. We describe the creation of a large data set of predictions from several smoothing algorithms about the acceptability of unseen grammatical phrases and a novel experimental method for the pairwise comparison of these models. We use this method to compare three smoothing methods and consider the results in light of the differences among the models. We argue that the data support the idea that similarity in this domain is best thought of as a form of asymmetric representational distortion and that the informational basis over which such estimates are made is broad, rather than narrow, as has been previously suggested.
\end{abstract}

The statistical technique of smoothing, which interpolates unknown values in a distribution, at once addresses twin problems faced by natural language processing (NLP) engineers and psycholinguists as a result of sparse data. Many NLP applications - for example, speech recognizers-rely on the assignment of occurrence probabilities. A spoken phrase is "recognized" as a function of the likelihood that it will occur, given the input speech signal. Ideally, likelihoods for occurrences of phrases are computed from the frequency of their occurrence in large corpora. However, the number of possible natural language constructions is so large that many plausible utterances fail to occur even in a very large corpus such as the World Wide Web. NLP engineers have used smoothing to estimate occurrence probabilities for nonoccurring phrases (Chen \& Goodman, 1998).

By contrast, psycholinguists have been interested in the relative acceptability of phrases (e.g., Cowart, 1997; Schütze, 1996). This is particularly interesting in the case of phrases that have failed to occur in the personal corpus of a given speaker's experience. In the following example, most native speakers of English would judge Statement 1a as being more plausible than Statement $1 \mathrm{~b}$, even if they have experienced neither statement previously:

(1a) High winds and strong currents made navigation difficult.

(1b) High currents and strong winds made navigation difficult.
Much of the investigation of relative acceptability (sometimes referred to as gradient acceptability) has focused on differences in well-formedness resulting from the violation of syntactic or phonological constraints (Sorace \& Keller, 2005). Differences in acceptability have been attributed to violations of constraints on, for example, gapping (Keller, 2001), auxiliary selection (Sorace, 2000), competition between word order and accent placement in Greek (Keller \& Alexopoulou, 2001), and dark versus light $/ 1 /$ in English (Hayes, 2000). However, there has been little or no investigation of the fact that native speakers-of English at least-perceive some apparently grammatical and intelligible sentences to be better formed than others. The speech of children and adults who have learned English as a second language often contains such phrases, as well as grammatical errors of commission (see, e.g., Bowerman, 1982, 1996; Lord, 1979), and are easily identified as such by adult native speakers. For example, although Statement $2 \mathrm{a}$ is acceptable, Statement $2 \mathrm{~b}$-an example of speech from an adult who had learned English as a second language-is less so.

(2a) The conference was hosted by the university.

(2b) I was hosted by my cousin.

This gradience might be expected in the case of known phrases-that is, those that have previously been encountered: It would not be surprising to discover that very frequently encountered phrases were judged to be more acceptable than those that were only infrequently encoun- 
tered. However, gradience in acceptability is also seen in novel phrases - that is, those that have not been previously encountered. Whereas NLP engineers need to assign differential probabilities to unseen phrases, psycholinguists aim to discover why those same phrases are differentially acceptable. In other words, they aim to answer the question, Why do people judge one phrase to be more acceptable than another, even when both phrases are equally parseable and semantically transparent and they have no experience of either?

In this article, we suggest that the smoothing techniques used by NLP engineers approximate the mechanisms by which unseen phrases come to be differentially acceptable and that the differences between different smoothing algorithms may shed further light on these psycholinguistic processes. The value of this approach is that several smoothing methods that differ in relatively subtle ways can be used for performing the same task. Comparing predictions about psycholinguistic acceptability using human judgments will allow us to make strong conclusions about the processes underlying those judgments on the basis of the differences between models. More broadly, these and other NLP techniques provide a set of useful tools for making more detailed proposals concerning the psycholinguistic processes underlying grammaticality judgments. Through the use of these techniques, we make the assumption that, in general, more acceptable phrases are more likely to be encountered - that is, they are more probable. Thus, an estimated probability of occurrence for an unseen phrase approximates to its acceptability. There are two aims of this article: First, we describe a large data set of estimates of co-occurrence probabilities for adjectivenoun pairs that fail to do-occur in the British National Corpus (BNC; Burnard, 1995) using a variety of smoothing algorithms, including a novel network implementation. The primary purpose of these data is to facilitate the exploration of smoothing as a psycholinguistic process responsible for the differential acceptability of nonoccurring phrases and to facilitate the comparison of smoothing algorithms in ways that will shed light on the aspects of their operation that are germane to the explanation of psycholinguistic acceptability. Second, therefore, to illustrate the potential of the data set in these respects, we present a short series of experiments that utilize a novel methodology for comparing several smoothing algorithms' capacity to predict acceptability judgments. We begin with a more detailed description of smoothing algorithms.

\section{Smoothing Algorithms}

Statistical smoothing techniques assume that some proportion of any data set is taken up with noise. They attempt to reduce this proportion by adjusting individual values toward the true signal using some algorithm to determine the direction and magnitude of adjustment."In linguistic smoothing, this typically means adjusting the occurrence probabilities of unheard phrases upward from zero.

Early discounting approaches to linguistic smoothing estimated probabilities for unseen items by pretending that those items had in fact been encountered a small number of times. Occurrences of 0 may be changed to 1 , for ex- ample, thus increasing the overall number of occurrences in the corpus. The probability of zero-occurrence items is thereby increased by decreasing the probability of others (Katz, 1987). Although this method does at least assign some probability to nonoccurring items, it does not differentiate between acceptable and unacceptable absentees.

By contrast, distance-weighted averaging (DWA) methods are based on the assumption that the likelihood of encountering a particular phrase should depend not only on the interword transitional probabilities of that phrase, but also on those of a set of similar phrases (Lapata, Keller, \& McDonald, 2001; Lee, 1999). For example, suppose that a bigram $w_{1} w_{2}$ has never before been encountered. A smoothing algorithm will attempt to compute the probability of $w_{2}$ following $w_{1}$ by first defining a set of words similar to $w_{1}$ and then inspecting their co-occurrence statistics to determine how often $w_{2}$ follows members of this set. If $w_{2}$ often follows words similar to $w_{1}$, it is probably acceptable as a partner of $w_{1}$ as well.

DWA methods differ mainly in the way that this similar set is constructed-in particular, the way in which similarity is computed. (This is discussed in more detail below.) Automated comparisons of discounting approaches with DWA have shown DWA to have superior performance (Dagan, Lee, \& Pereira, 1999). We therefore end the discussion of discounting methods here (but see Chen \& Goodman, 1998, for a review). The following section discusses DWA methods in more detail and with particular reference to adjective-noun pairs, which provide a straightforward means of comparing these mechanisms. We also introduce a novel smoothing mechanism - an associative smoothing network (ASN). This method is based on the same intuitions that underpin DWA but has several advantages of computational simplicity.

\section{DWA and Distributional Similarity}

To investigate smoothing as a psycholinguistic process, we made use of adjective-noun co-occurrence statistics from the BNC. The main reasons for this decision were (1) precedent-previous work has validated smoothing algorithms using adjective-noun pairs (Lapata et al., 2001; Lapata, McDonald, \& Keller, 1999), and (2) that this construction has the virtue of extreme simplicity and thus facilitates extraction from corpora and subsequent processing.

To estimate the probability $\hat{P}(n \mid a)$-that an unseen (one that does not occur in the BNC) adjective-noun pair $a, n$ will occur, the co-occurrence statistics for $a$ and $n$ are used to find a set of adjectives similar to $a$. (Details of how similarities are computed are described below.) The estimate is then based on the co-occurrence statistics of this set:

$$
\hat{P}(n \mid a)=\frac{\sum_{b \in S_{a}} \operatorname{sim}(a, b) P(n \mid b)}{\sum_{b \in S_{a}} \operatorname{sim}(a, b)},
$$

where $b$ is a member of $S_{a}$, the set of adjectives similar to $a$, and $\operatorname{sim}(a, b)$ is the similarity between adjectives $a$ and $b$ (Lee, 1999). A nonoccurring adjective-noun pair $a, n$ is deemed plausible if $\boldsymbol{n}$ has occurred often with a number of adjectives similar to $a$. The major difference between the 
DWA algorithms that have been proposed lies in the way in which similarities between adjectives are computed.

Class-based smoothing determines the similarity of two words by considering the word classes to which they both belong (Lapata et al., 2001). Classes may be algorithmically derived from a corpus or taken from a manually constructed taxonomy such as WordNet (Miller, Beckwith, Fellbaum, Gross, \& Miller, 1990). By contrast, distributional similarity techniques compute similarity as a function of the collocational statistics of the adjectives and nouns involved. Class-based smoothing that bases similarity on shared senses in WordNet has been shown to correlate slightly better with acceptability judgments than do some forms of distributional similarity methods (Lapata et al., 2001). This should not be surprising. The use of classes, whether derived from a corpus or manually constructed, entails an additional level of information over raw collocational statistics. It also seems likely that, in constructing a taxonomy of classes, people may well be using many of the same aspects of judgment that are used to evaluate the acceptability of a novel phrase. Thus, the finding that manually constructed classes provide a good basis for acceptability judgments may be circular. It is certainly possible to derive classes algorithmically from collocational statistics, but it is not clear that this offers any benefit over distributional similarity measures and is less theoretically parsimonious. We therefore concentrate on DWA methods involving distributional similarity measures. Note that our argument is not that classes cannot be involved. Rather, distributional similarity measures are theoretically more parsimonious, and the transparency of operation of distributional statistical methods will offer greater insight into the psychological mechanisms involved.

We now turn to ways in which distributional similarity may be computed in DWA algorithms. Adjectives $a$ and $b$ (Equation 1) are represented as vectors, where each point is a probability derived from the number of times the adjective has co-occurred with a particular nounspecifically, the probability of $n$ given $a$ is the number of times that $n$ has followed $a$, divided by the total number of times that $a$ has occurred. The similarity between $a$ and $b$ is the similarity between these two vectors, and this may be defined in several ways. A common definition of distributional similarity is as a function of the distance between the points in multidimensional space defined by the vectors in question-for example, Euclidean (EUC; a common measure of similarity in psychology), cosine, or city-block distances. In contrast, Lee (1999) identifies an alternative class of similarity algorithms that rely on set theoretic notions of similarity. Specifically, $a$ and $b$ may be thought of as being represented by sets of nouns-that is, all the nonzero points in the co-occurrence vector. It is, then, the intersection $a \cap b$ that determines $\operatorname{sim}(a, b)$ most effectively. Lee describes two similarity functions that rely on this set: Jaccard's coefficient (JAC) and the skew divergence (SKEW), which, according to Lee, is "an approximation to the KL divergence [the Kullback-Leibler divergence; see below] to be used when sparse data problems would cause the latter measure to be undefined" (p. 30). As she points out, this is strictly a dissimilarity measure.
However, it can easily be recast as a similarity function by subtracting from an appropriate constant, taking reciprocals, or by numerous other means.

Jaccard's coefficient is a set theoretic measure that operates only on the nonzero elements of the vectors and ignores their actual values:

$$
\operatorname{JAC}(a, b)=\frac{|a \cap b|}{|a \cup b|} .
$$

So simply knowing the size of the intersection of supports relative to the size of their union provides a good measure of distributional similarity. The $\mathrm{KL}$ divergence, $D$, is an information theoretic measure determining inefficiency in coding one distribution using the other:

$$
D(P \| Q)=\sum_{n} P_{n} \log \frac{P_{n}}{Q_{n}},
$$

where $n$ are the nouns with which adjectives $a$ and $b$ cooccur, and $P$ and $Q$ are the probability distributions of $n$, given $a$ and $b$, respectively. Note that the KL divergence is undefined if any point $(n \mid a)$ or $(n \mid b)$ is zero. It cannot, therefore, determine the similarity between co-occurrence or probability vectors which contain nonoccurring pairs without modification. One such change is the JensenShannon divergence, of which Lee's solution-the skew divergence-is an asymmetric generalization:

$$
D_{\alpha}(P, Q)=D[Q \| \alpha \cdot P+(1-\alpha) \cdot Q],
$$

where $0 \leq \alpha \leq 1$ can be thought of as the degree of confidence in the distribution $a$. It is also worth noting that this function fulfills the asymmetry criterion for similarity functions: It is not necessarily true that word $i$ is as similar to word $j$ as word $j$ is to $i$. For example, bird may be a better substitute for duck than duck is for bird. Tversky (1977) gives many more such examples.

Below we describe the computation of estimates for nonoccurring adjective-noun pairs with DWA using three similarity measures. These are EUC, JAC, which has the computational advantage of extreme simplicity, and SKEW, which Lee (1999) reports as providing superior probability estimates on an automated completion task. First, however, we introduce one further smoothing mechanism: the ASN. This is a novel method of smoothing based on simple associative principles.

\section{The Associative Smoothing Network (ASN)}

The ASN can be thought of in terms of spreading activation. When an adjective is activated, say real, activation spreads to all the nouns with which real is associated (i.e., all those with which it has co-occurred; for example, people and time). From these nouns, activation then spreads back to the other adjectives that are associated with all those nouns (e.g., good). Finally, activation spreads once more from this set of adjectives to the nouns with which all of them have co-occurred (e.g., students). This process is illustrated in Figure 1.

Activation in the ASN spreads by matrix multiplication. If we have $A$ adjectives and $N$ nouns, the adjecive-noun co-occurrence statistics can be contained in a matrix $M$ 
with dimensions $A$ and $N$. Adjective and noun activations can be expressed as vectors $V_{a}$ and $V_{n}$, of sizes $A$ and $N$, respectively. Spreading activation from adjectives to nouns is simply a matter of multiplying the vectors with the matrix; activation in $V_{n}$ is the product of $V_{a}$ and $M$, whereas activation in $V_{a}$ is the product of $V_{n}$ and $M$.

Thus far we have implied that $M$ contains raw cooccurrence statistics. This is a possibility, but it is equally plausible that activation could be spread from $V_{a}$ to $V_{n}$ and back by some function of frequencies of pairs. Whilst there are theoretically infinitely many candidate functions, it is in keeping with the simplicity of the underpinning principles of the ASN to deviate as little as possible from raw co-occurrence values. We therefore make only the standard assumption that perceived values are logarithmically related to actual values (see, e.g., Lapata et al., 2001; Lapata et al., 1999).

This mechanism has several virtues over the variations on DWA described above: It is based on psychologically plausible associative principles. It is more parsimonious; DWA models require the set of similar neighbors to be restricted to a fraction of those available (Lee, 1999, and see below). In contrast, the ASN performs at its best on the same automated task when all the available neighbors are included (Roberts, 2005), thus enabling us to abandon the $k$ parameter for the ASN. And, on a practical note, it operates by spreading activation between vectors. This requires only simple matrix multiplication and is therefore more efficient than the explicit similarity computations described above.

\section{Differences in Optimal Information Use Between the ASN and DWA}

An important parameter of any DWA algorithm is $k$, the size of the set $S_{a}$ of adjectives similar to $a$ (see Equation 1). Lee (1999) reports that, to perform optimally in an automated completion task, DWA algorithms required this set to be limited to $10 \%$ of the total number of adjectives available. She compared a number of DWA methods using verb-object pairs. The task was as follows: Counts for verb-object (i.e., verb-noun) pairs were extracted from a corpus. Twenty percent of the pair tokens were removed, and any type whose count was reduced to zero was used as a test item. Each test pair was then associated with a second noun, and the algorithm estimated the probability of each of the two nouns given the test verb. It was counted as being correct if the probability was higher for the genuine object. As $k$, the number of similar items, increased from $10 \%$ to $100 \%$ of the possible members of $S_{a}$, rates of error rose from around $26 \%$ to nearly $40 \%$. To compare the influence of $k$ on DWA and on the ASN, we first extracted co-occurrence statistics for the 1,000 most frequent adjectives and 1,000 most frequent nouns in the BNC. We then ran Lee's automated completion task for DWA $_{\mathrm{JAC}}$ and the ASN with $k$ set at $10 \%$ intervals of all of the available candidates. For the ASN, this meant that at



real
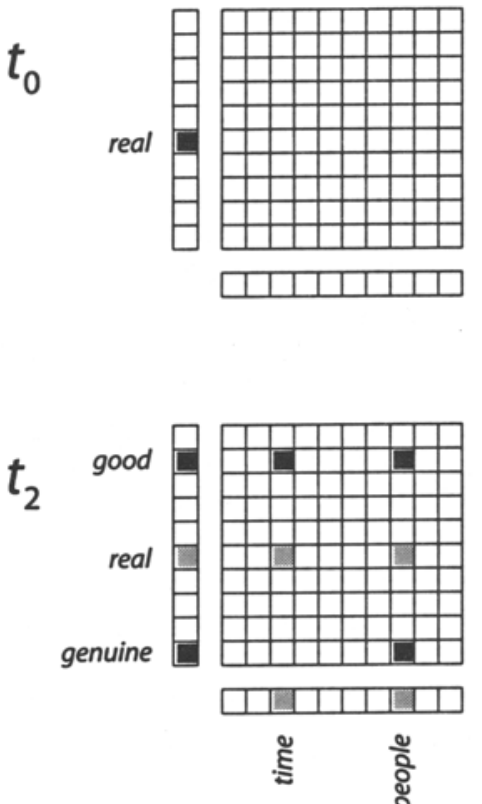

$t_{1}$

real
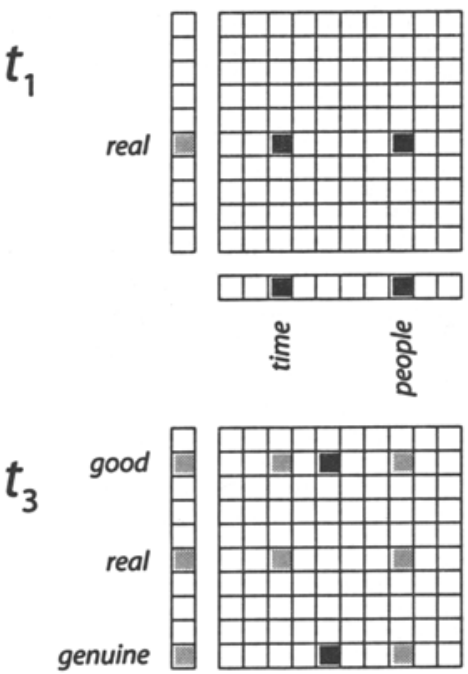

हั

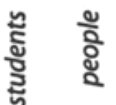

Figure 1. The operation of the ASN. An adjective (real) is activated at time $t_{0}$. At $t_{1}$, activation is spread to nouns by multiplying the adjective activation vector through the weights matrix. This produces activation in all the nouns with which real has co-occurred. At $t_{2}$, activation is spread from these nouns back through the weights matrix in the same manner, activating a set of adjectives. These are similar to real in that they have occurred with some of the same nouns.At time $t_{3}$, activation is again spread to the nouns. In this manner, predictions are made about phrases that have not occurred: The phrase real students does not occur in the British National Corpus. 
time $t_{2}$ (Figure 1), only the $k$ largest activations were fed forward to the noun vector at time $t_{3}$; the remainder were reset to zero. The test was run 100 times at each level for each algorithm. The means and standard deviations for each level are shown in Figure 2.

The size of $S_{a}$ is not a natural parameter for the ASN; some violation of the simple associative principles of the network is required to incorporate it. These data show that the ASN differs qualitatively from DWA methods in that it approaches optimal performance when all the available data are used. This represents a potentially useful difference between the ASN and DWA methods. Since the two mechanisms are similar in many respects, this is likely to be one of the major sources of any difference in terms of their fit to native speaker intuitions of well-formedness. In what follows, $k$ is set to $10 \%$ of the similar items available for all of the DWA methods; the ASN incorporates no $k$ parameter. The $1,000 \times 1,000$ matrix used in these tests is probably not appropriate for estimation of human acceptability judgments, however; vocabularies of native speakers are, of course, much larger. We now describe the construction of a very much larger data set of estimates.

\section{Construction of a Large Data Set of Adjective-Noun Co-Occurrence Estimates}

The initial decision in the construction of the data set was the number of adjectives and nouns to be included. Aside from the computational difficulties involved in including every word tagged as an adjective or noun in the $\mathrm{BNC}$, it is probably not psychologically realistic to expect that every word in a static corpus will contribute. We therefore used lexical decision data for words occurring

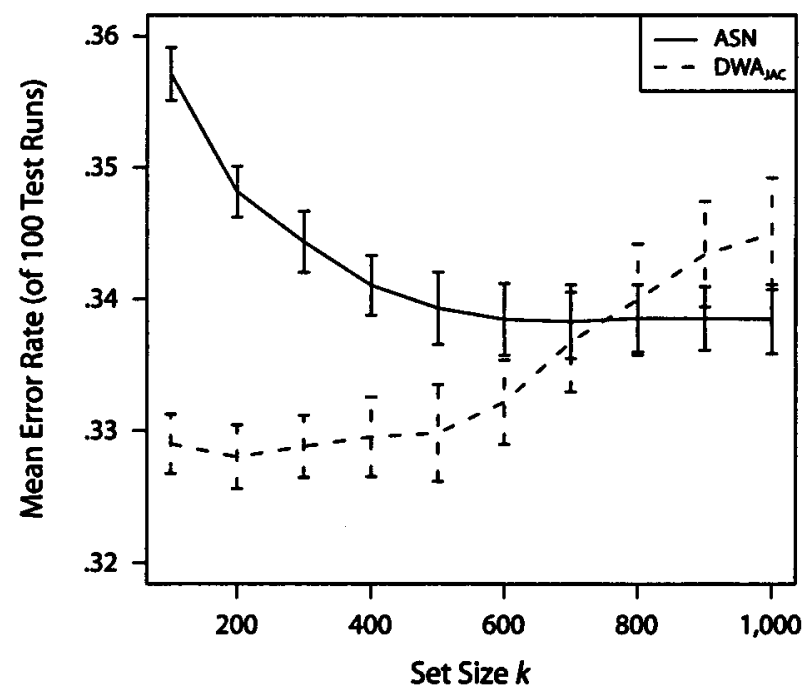

Figure 2. Mean error rates and standard deviations for the ASN and DWA $\mathrm{JAC}_{\mathrm{JC}}$ on the automated completion task. Set size $k$ is the size of the comparison set on which likellhood estimates were based. Note that $k$ has subtly different meanings for DWA $A_{\text {IAC }}$ and the ASN. For DWA JAC, $_{\text {, }}$ it refers to the number of similar adjectives used to compute smoothed probabilities. For the ASN, it refers to the number of adjective activations not reset to zero at time $t_{2}$ (Figure 1). in the BNC collected by Adelman and Brown (2008) to determine a "cutoff" frequency that corresponds approximately to the point at which participants' responses about whether a particular word was real or not were at chance. In other words, we excluded words that were more likely than not to be judged as being nonwords.

The method of determining this point was as follows: Each word for which lexical decision data were available, irrespective of class, was associated with its BNC frequency from the unlemmatized lists made available by Kilgarriff. (At the time of writing, they are downloadable from www.kilgarriff.co.uk/bnc-readme.html.) Words were then ranked according to frequency, and a mean lexical decision score was associated with each word. This was the mean lexical decision score for the word itself and the following 99 words - that is, a window of 100 words. A mean score of .5 was found to be associated with a frequency of 50 in the whole corpus, or approximately 1 per 2 million. Words with this frequency or greater are likely to be on the cusp of participants' lexicons and therefore contribute minimally to their lexical processing-for example, by competing only weakly for recognition.

We extracted from the BNC 6,381 words that were tagged unambiguously as adjectives and 25,491 words tagged unambiguously as nouns, all of which had a corpus frequency of equal to or greater than 50 . Words containing nonalphabetic characters such as hyphens were excluded. This yielded a co-occurrence space of $162,658,071$ pairs. We searched the BNC for occurrences of these pairs using a simple collocation search: Only occurrences of the form

$$
\underset{<\text { noun }^{<}}{<\text {ajective }_{0}>\ldots<\text { adjective }_{i}>\ldots<\text { adjective }_{n}>}
$$

were extracted, with each adjective ${ }_{i}$ being associated with the terminal noun. Clearly, this excludes remote pairings, as in, "The sun was by this time rather warm," and in this respect differs from previous work (Lapata et al., 2001). The principal reason for this decision relates to our experimental method described below. The principal purpose of these estimates is to facilitate investigation of smoothing as a psychological mechanism, and, to this end, we aim to compare smoothing estimates against acceptability data collected from human participants. In our experimental evaluations, we maintained consistency and simplicity in our stimuli by restricting sentences to those containing proximal adjectival noun phrases-that is, those of the form < adjective> <noun>-and based our smoothing estimates on co-occurrences of the same form.

This issue raises an obvious concern about the level at which smoothing takes place; at a semantic or categorical level, one might expect remote adjective-noun pairs to influence the acceptability of proximal ones. At the level of surface distributional statistics, one might expect that they would not. Thus, a second reason for excluding remote pairings is that it is surface statistical information on which linguistic smoothing relies. Nonctheless, we acknowledge that it remains an open empiricai quesiion whether remote pairs influence the acceptability of proxi- 
mal ones, but addressing that question is beyond the scope of this article.

The extraction yielded a total of $1,129,923$ pairs occurring between 1 and 9,463 times each. It is noteworthy that this covers less than $1 \%$ of the possible space of cooccurrences; the proportion of plausible-but-unheard sentences in the whole of English is presumably considerably smaller than this. These co-occurrences were represented in a matrix of adjectives by nouns. From this matrix we computed a matrix of co-occurrence probabilities where the probability of an adjective-noun pair $a, n$ was:

$$
P(a, n)=\frac{f r e q(a, n)}{\sum_{i}^{N} \text { freq }\left(n_{i} \mid a\right)},
$$

where freq $(a, n)$ is the corpus frequency of $a, n$ and $N$ is the total number of nouns represented in the matrix. Thus, $\Sigma_{i}^{N}$ freq $\left(n_{i} \mid a\right)$ is the total number of times that $a$ has occurred as part of an adjective-noun pair. Note that this entails the assumption of left-to-right transitional dependency, although, at another level of abstraction, one might make the opposite assumption. For example, at the level of semantics, one might assume that the noun is primary and compute the probabilities of particular adjectives modifying individual nouns.

This matrix was then used to compute smoothed probabilities for each of the nonoccurring pairs. Each adjective is represented in the matrix as a vector of probabilities. Pairwise similarities were computed between the probability distributions representing each adjective and that representing every other adjective, using EUC, JAC, and SKEW. These similarities were then used to compute three DWA estimates for the co-occurrence probabilities of each nonoccutring adjective-noun pair. In addition, a similar set of estimates was computed using the ASN. This computation was based on the log values of raw co-occurrence counts rather than on probabilities (see above). The ASN estimates were therefore not co-occurrence probabilities, but noun vector activations at time $t_{3}$ (see Figure 1). Nonetheless, they are cardinal data sufficient for quantitative comparison with the probability estimates provided by the DWA algorithms; in addition, conversion to probabilities would be straightforward.

Finally, for each of these estimates, the centile rank was estimated by determining their rank in relation to a sample of 1,000 randomly selected estimates of the same type. This allowed for broad comparisons of rank without the need for precise sorting of upward of 162 million items.

The principal components of the data set are, then, (1) a matrix of BNC co-occurrences for 6,381 adjectives and 25,491 nouns unambiguously tagged as such, containing only alphabetic characters, and with a corpus frequency of at least 50; (2) three similarities (EUC, JAC, and SKEW) of the adjective to the other adjectives, on the basis of these co-occurrences; ( 3 ) four smoothed estimates for the likelihood or acceptability of all of those pairs that fail to occur in the BNC; and (4) for each estimate of acceptability, an estimate of its centile rank. We will now describe a novel method for using the data as the basis for experimental comparison.

\section{EXPERIMENTAL COMPARISON OF SMOOTHING ALGORITHMS}

We present a pairwise comparison of the following three of the four smoothing algorithms described above: $\mathrm{DWA}_{\mathrm{EUC}}, \mathrm{DWA}_{\mathrm{SKEW}}$ and the ASN. We have not included $D_{W A} A_{\text {IAC }}$ in this set because previous work has shown that its performance extremely closely resembles that of DWA $_{\text {SKEW }}$ (Lee, 1999) and that the variance captured by it in terms of human acceptability judgments may, in fact, be a subset of that captured by both DWA SKEW $_{\text {and the ASN }}$ (Roberts, 2005). Since its inclusion would double the number of comparisons to report and would likely add little to the discussion, we have omitted it for the sake of brevity.

We first compare the new ASN with DWA SKEW $_{\text {and }}$ DWA $_{\text {EUC. }}$. We then compare the two DWA algorithms with each other. We find that, although there is considerable overlap between all three, there are double dissociations between the ASN and each of the DWA methods. For the first two pairwise comparisons, in other words, each smoothing method predicts elements of the variance of human performance not predicted by the other. However, the third comparison-that between the two DWA methods-suggests that predictions from DWA EUC $_{\text {may, in fact, }}$ be wholly or mainly a subset of those of DWA SKEW.

\section{Experiment 1 \\ ASN Versus DWA}

In Experiment 1, we compared acceptability predictions from the novel ASN with those of DWA SKEW. $_{\text {. }}$

\section{Method}

The two smoothing algorithms were compared using a withinsubjects design. On each trial, participants selected the more acceptable of two sentences. Sentences were always of the form "The $<$ adjective $><$ noun $><$ tense $><$ verb $>$," where <tense $>$ was substituted for one of was, will be, may have been, or might be, and <verb > was substituted for one of discussed, mentioned, or talked about. Each pair of sentences contained the same <tense $>$ and <noun> substitutions but differed in their <adjective $>$ and $<$ noun> substitutions, as in the example below:

(3a) The intolerable mediocrity will be discussed.

(3b) The unequivocal tsarist will be discussed.

Each experiment comprised two conditions, with each condition testing the ability of one smoothing algorithm to account for aspects of acceptability judgments not accounted for by the other. In the first condition, the ASN predicted a large difference in acceptability between the two sentences, whereas DWA SKEw $_{\text {predicted no difference. }}$ In the second, DWA ASN predicted none. Thus, in each condition, responses deviating systematically from chance indicated that one algorithm captured a difference in acceptability not captured by the other.

Participants. The participants were 28 undergraduate students (18 females, mean age $21.1 ; 10$ males, mean age 19.8) at the University of Warwick, each of whom received $£ 2$ for participating.

Stimuli. Each participant completed 100 trials ( 50 per condition). Stimuli were selected using the following criteria: We used the rank centile estimates to extract the most acceptable $10 \%$ of adjectivenoun pairs according to each of the two smoothing algorithms under 
consideration (hereafter acceptable), and all those predicted by each algorithm to have zero acceptability (hereafter unacceptable). Thus, four sets in all were extracted. Three intersections between these four sets were then found: (1) those pairs acceptable according to the ASN and unacceptable according to DWA ceptable according to DWA SKEW $_{\text {and }}$ unacceptable according to the ASN; and (3) those unacceptable to both the ASN and DWA SKEW $_{\text {. }}$ Stimulus sets for the two conditions were then constructed by pairing members of the first two intersections (acceptable only to one algorithm) with members of the third (unacceptable to both). Statement 3a, then, might be acceptable to the ASN but unacceptable to

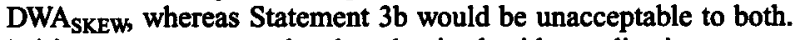
Pairings were automated and randomized, with no adjective or noun used more than once.

Procedure. Participants were seated in front of a computer screen in a cubicle. They were told that the experiment was about their intuitions as native speakers of English and that they would be presented pairs of sentences. They were asked to imagine that one sentence had been spoken by a monolingual native speaker of English, the other by someone who had learned English as a second language, and that the task was to indicate which sentence had been spoken by the native speaker of English. Pairs of sentences such as those in Example 3 were presented bilaterally on the screen in random order. The left/right location of sentences was randomized for each participant. Participants indicated the sentence they thought more likely to have been produced by a native English speaker by clicking on it with a mouse.

\section{Results}

In Condition 1, the ASN predicted a large acceptability difference between the two sentences, whereas DWA SKEW $_{\text {S }}$ predicted that both sentences would be found equally (and strongly) unacceptable. Responses corresponding with the sentences predicted by the ASN to be found more acceptable scored a 1 , and those corresponding with less acceptable sentences scored a 0 . Thus, a score greater than .5 indicates that the ASN predicts aspects of acceptability


reversed, with DWA SKEW $_{\text {predicting a large difference in }}$ the acceptability of the two sentences and the ASN predicting that they are both equally and strongly unacceptable. In this condition, responses corresponding with the sentences predicted by DWA SKEW $_{\text {to }}$ be found more acceptable scored a 1, and those corresponding with less acceptable sentences scored a 0 . A score significantly greater than .5 in Condition 2 indicates that DWA SKEW $_{\text {predicts elements }}$ of acceptability that are not predicted by the ASN.

The mean score in Condition 1 was .64; this was strongly significant $[t(27)=10.60, p<.001$, two-tailed $]$. Thus, the ASN predicts differences in acceptability that

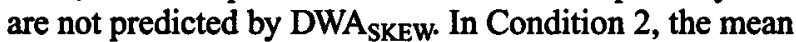
score was .59; this too was strongly significant $[t(27)=$ $5.31, p<.001$, two-tailed]. DWA SKEW $_{\text {can }}$ therefore be said to predict differences in acceptability that are not predicted by the ASN. In addition, the difference between the two conditions is shown to be significant [two-sample test: $t(27)=2.9, p<.05]$. Thus, significantly more differences are predicted by the ASN but not DWA SKEW $_{\text {than }}$ by DWA

It is important to clarify these results in terms of their implications for the differences between the two algorithms, since the interpretation is, perhaps, unusual. The main differences between the ASN and DWA SKEW $_{\text {are cap- }}$ tured by the two separate condition means; these figures indicate the degree to which each model captures variance that is not captured by the other. We will later interpret these differences in terms of different features of the models. The difference between the two condition means offers a way to assign relative weight to those features: The significantly greater portion of the variance captured by the ASN can be thought of as reflecting the relatively greater importance of its unique features. It is also worth noting that, although the difference between the conditions is not large in absolute terms ( $5 \%$ of the range $0-1)$, we are interested in differences from chance. Viewed in this way, the ASN predicts approximately $56 \%$ more of the acceptability difference than does DWA

These data show a double dissociation between the ASN

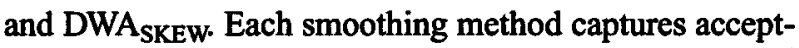
ability differences between sentences that are not captured by the other. This suggests that the individual elements of each method describe separate contributions to sentence acceptability. However, the number of differences captured uniquely by the ASN is significantly higher than the number captured uniquely by DWA $_{\text {SKEW, }}$ suggesting that the unique elements of the ASN describe a greater contribution. These contributions are discussed below.

\section{Experiment 2 ASN Versus DWA Euc}

In Experiment 2, we compared acceptability predictions from the novel ASN with those of DWA $\mathrm{EUC}_{\text {. }}$

\section{Method}

The experimental method and procedure were identical to those of Experiment 1. The stimuli were selected in the same manner as in Experiment 1, except for the substitution of DWA ${ }_{E U C}$ for DWA SKEW. $_{\text {. }}$

Participants. The participants were 30 undergraduates from the University of Warwick (22 female, mean age $21.1 ; 8$ males, mean age 20.8), each of whom received $f 2$ for participating.

\section{Results}

In Condition 1, the ASN predicted a large acceptability difference between the two sentences, whereas DWA EUC predicted that both sentences would be found to be equally (and strongly) unacceptable. Responses corresponding with the sentences predicted by the ASN to be found more acceptable scored a 1 , and those corresponding with less acceptable sentences scored a 0 . Thus, a score greater than .5 indicates that the ASN predicts aspects of acceptability

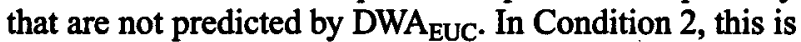
reversed, so that a score significantly greater than .5 in Condition 2 indicates that DWA EUC $_{\text {predicts elements of }}$ acceptability that are not predicted by the ASN.

The mean score in Condition 1 was .63 , which is significantly different from $.5[t(29)=9.45, p<.001$, two-tailed]. Thus, the ASN predicts many acceptability differences between sentences that are not predicted by

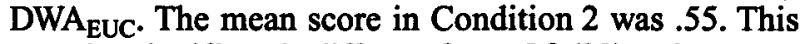
was also significantly different from $.5[t(29)=3.79, p<$ $.001]$. Thus, DWA $\mathrm{EUC}_{\mathrm{C}}$ also predicts several differences in sentence acceptability that are not predicted by the ASN. The difference between the two conditions was also significant [two-sample test: $t(29)=5.0, p<.001$, two-tailed]. 
It is interesting to note the high similarity between the results of Experiments 1 and those of Experiment 2. In both cases, both the ASN and the DWA algorithms captured aspects of acceptability differences that were not accounted for by the other. Also in both cases, the ASN captures significantly more of these differences than the DWA algorithms. The similarity between these results requires that we investigate the extent of the similarity between the two DWA algorithms. Although they show marked differences on Lee's (1999) automated comparisons, it is nonetheless the case that their predictions are strongly correlated $(r=.67$; Roberts, 2005). It may therefore be the case that, when differences that can be accounted for by the ASN are controlled for, there is no significant difference between the predictions made by the two DWA algorithms. In Experiment 3, we directly compared DWA EUC $_{\text {with }}$ DWA tent to which this is the case.

\section{Experiment 3 DWA EuC Versus DWA SKEW}

\section{Method}

The method and procedure employed were again identical to those of Experiment 1. Stimuli were again selected as for Experiment 1, this time substituting the two DWA algorithms.

Participants. The participants were 26 undergraduates from the University of Warwick (19 females, mean age 22.4; 7 males, mean age 20.6), each of whom received $£ 2$ for participating.

\section{Results}

In Condition 1, DWA EUc $_{\text {predicted a large acceptability }}$ difference between the two sentences, whereas DWA predicted that both sentences would be found to be equally (and strongly) unacceptable. Responses corresponding with the sentences predicted by the DWA $\mathrm{EUC}_{\mathrm{C}}$ to be more acceptable scored a 1 , and those corresponding with less acceptable sentences scored a $\mathbf{0}$. Thus, a score greater than .5 indicates that the DWA $\mathrm{EUC}_{\mathrm{C}}$ predicts aspects of acceptability that are not predicted by DWA tion 2, DWA SKEW $_{\text {predicted a large difference in the ac- }}$ ceptability of the two sentences, and the ASN predicted that they would be found to be equally (and strongly) unacceptable. In this condition, responses corresponding with the sentences predicted by DWA SKEW $_{\text {to }}$ be more acceptable scored a 1 , and those corresponding with less acceptable sentences scored a 0 . A score significantly greater than .5 in Condition 2 indicates that DWA predicts elements of acceptability that are not predicted by the DWA EUC. $_{\text {. }}$

In Condition 1, the mean score was .53. This was not significantly different from chance. In Condition 2 , the mean score was .64 . This differed significantly from .5 $[t(25)=10.48, p<.001$, two-tailed]. Thus, DWA skew appears to predict all the differences that are predicted by $\mathrm{DWA}_{\mathrm{EUC}}$, as well as some that are not.

\section{DISCUSSION}

In comparing the ASN with each DWA method, we found evidence that suggests that each algorithm predicted acceptability differences that were not predicted by the other, but that in each case, the ASN predicted significantly more than did either DWA algorithm. In comparing the two DWA algorithms, it was found that,

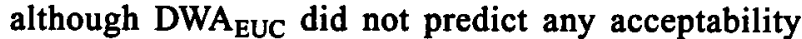
differences that were not also predicted by DWA DWA $_{\text {SKEW }}$ did predict differences that were not predicted by DWA

The only difference between DWA is the way in which the similarity between co-occurrence vectors is computed. DWA EUC $_{\text {assumes similarity to be a }}$ function of the spatial distance between vectors (Shepard, 1957). DWA SKEW assumes that similarity is a function of the difficulty of transforming one into another-strictly, the average inefficiency of using one to code another-a view of similarity suggested by Hahn, Chater, and Richardson (2003). The comparison of the two DWA methods suggests that the spatial distance view produces acceptability estimates that are a subset of those produced by the representational distortion view. We conclude that, at least in the case of the acceptability of novel lexical combinations, similarity is better thought of as being a form of representational distortion than of physical distance.

Given this finding, we will collapse the comparison of the ASN with the two DWA methods and discuss only Experiment 1, the comparison with DWA comparison of DWA methods, the strength of this experiment is that there are considerable conceptual similarities between the two smoothing algorithms. The presence of small differences enables relatively strong conclusions to be drawn from the results. There are two substantial differences between the two methods: (1) the informational basis upon which estimates are made-DWA SKEW $_{\text {S }}$ requires only a relatively small number of similar adjectives to be used to make optimal estimates (at least in the automated comparison), whereas the ASN makes use of all the similarity information available; and (2) the way in which similarity is computed.

Although the ASN does not compute similarity explicitly, we may nonetheless infer several points about the way in which it is computed implicitly from the distribution of activation in the sets of similar adjectives that are produced at $t_{2}$ and from the action of the network. Nowhere does the ASN compute a figure defining the similarity between two adjectives; but, nonetheless, a set of adjectives similar to a target adjective is produced at $t_{2}$. This set can be thought of as being equivalent to $S_{a}$, the set of adjectives similar to $a$ that is computed by DWA methods. It should be clear from Figure 1 that the ASN embodies a view of similarity that corresponds to the notion of common and distinctive features (Tversky, 1977). Adjectives are similar to the degree that they have previously co-occurred with the same nouns. Lee (1999) states that it can be shown that the skew divergence also relies on this set of common co-occurrences, but that it is asymmetric: The similarity of long to tall will not necessarily be the same as that for tall to long, or, more formally, $D_{a}$ (tall $\|$ long $) \neq D_{\alpha}($ long $\|$ tall $)$. There is no corresponding asymmetry in the ASN. If the model is computing smoothed noun activations for tall, and long receives $P$ units of activation at $t_{2}$, then tall will also re- 
ceive $P$ units from long at $t_{2}$ when the model is computing smoothed noun activations for long. Thus, the major difference between their approaches to the similarity of the distributed representations of words is the asymmetry of the skew divergence, as opposed to the implied symmetry of the ASN.

The ASN predicted a large number of differences that were not predicted by DWA SKEW. We interpret this as resulting from the difference in amount of information utilized by the two algorithms-specifically, the fact that the ASN makes use of all of the available informa-


DWA ${ }_{\text {SKEW }}$ also predicted a significant number that were not predicted by the ASN. We interpret this as resulting from the difference in approaches to similarity between the two methods-specifically, that the skew divergence naturally embodies the asymmetry of similarity (Tversky, 1977), whereas the ASN does not.

This interpretation has several implications for future research. First, it may be the case that DWA be improved simply by utilizing all of the information available. Were this the case, it would suggest that the automated completion task is, in fact, antithetical to the evaluation of smoothing algorithms as proxies for human intuitions about acceptability. However, it may also be the case that the use of the maximum number of similar adjectives interacts with the algorithm, so that it is optimal for the ASN, but not for DWA, as is suggested by the results of the automated task above. Testing these hypotheses would involve recomputing the set of predictions from

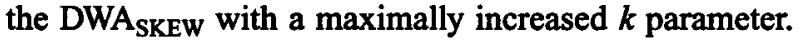
One could then attempt to find a set of stimuli with which to compare the two sets of predictions from DWA is possible that there would then be insufficient overlap between the maximally and minimally acceptable predictions to effect such a comparison; relaxing the stringency of what counts as acceptable or unacceptable could resolve this, but would reduce the directness of comparison with the present findings.

Second, this interpretation suggests that similarity is best viewed, in this case at least, as being asymmetric representational distortion. As noted above, asymmetry is in any case an observable and intuitive feature of similarity (Tversky, 1977), and its presence in the skew divergence makes it an obvious candidate for explaining the acceptability variance captured uniquely by that measure. Nonetheless, it would be useful to explore it more explicitly and to unconfound it from the differences in the informational bases of the two smoothing methods compared here. Jaccard's coefficient presents a candidate function that makes explicit the dependence on overlapping features implied by the ASN. As noted above, previous work has suggested that the variance captured by DWA $_{\mathrm{JAC}}$ is, in fact, a subset of that captured by $D W A_{\text {SKEW, }}$ similar to the way in which the results

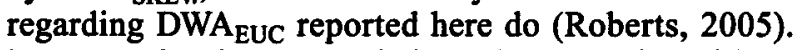
However, the above speculations about the size of $k$ relate as much to DWA $\mathrm{JAC}_{\text {ac }}$ as they do to DWA findings regarding the optimal size of $k$ for DWA should therefore be transferred to $\mathrm{DWA}_{\mathrm{JAC}}$, and the comparison repeated.

In this article, we have described the requirement for an account of differences in acceptability between unseen but nonetheless grammatical phrases for native speakers. We suggested that the technique of smoothing might be used as a candidate mechanism whose investigation may shed light on the process. The creation of a large data set of smoothed estimates was described, as was a novel mechanism for comparing different smoothing algorithms. Three such methods were compared in a pairwise experimental manner, and the results were discussed in the light of key differences between the smoothing models. Variance uniquely predicted by DWA $_{\text {SKEW }}$ was interpreted as indicating that similarity in this case is best viewed as an asymmetric form of representational distortion, whereas variance uniquely predicted by the ASN was interpreted as indicating that the requirement that the size of $k$ be minimized may be misplaced. We have suggested several lines of research by which these issues may be further explored.

\section{AUTHOR NOTE}

Bothauthors were supported by ESRCGrant NumberRES-000-22-1120. M.A.J.R. received partial support from ESRC Grant Number R39942. N.C. was further supported by a Major Research Fellowship from the Leverhulme Trust. Correspondence concerning this article should be addressed to M. A. J. Roberts, Department of Psychology, University of Edinburgh, Edinburgh EH8 9JZ, Scotland (e-mail: matthew.roberts@ ed.ac.uk).

\section{REFERENCES}

Adelman, J. S., \& Brown, G. D. A. (2008). Modeling lexical decision: The form of frequency and diversity effects. Psychological Review, 115, 214-229.

BoWERMAN, M. (1982). Evaluating competing linguistic models with language acquisition data: Implications of developmental errors with causative verbs. Quaderni di Semantica, 3, 5-66.

BoWERMAN, M. (1996). Argument structure and learnability: Is a solution in sight? Proceedings of the Berkeley Linguistics Society, 22, 454-468.

BURNARD, L. (1995). User's reference guide for the British National Corpus. Oxford: Oxford University Computing Service.

CHEN, S. F., \& GoOdMAN, J. (1998). An empirical study of smoothing techniques for language modeling (Tech. Rep. No. TR-10-98). Cambridge, MA: Harvard University, Center for Research in Computing Technology.

COWART, W. (1997). Experimental syntax: Applying objective methods to sentence judgments. Thousand Oaks, CA: Sage Publications.

Dagan, I., LeE, L., \& PerRira, F. C. N. (1999). Similarity-based models of word cooccurrence probabilities. Machine Learning, 34, 43-69.

Hahn, U., Chater, N., \& Richardson, L. B. (2003). Similarity as transformation. Cognition, 87, 1-32.

HAYES, B. P. (2000). Gradient well-formedness in optimality theory. In J. Dekkers, F. van der Leeuw, \& J. van de Weijer (Eds.), Optimality theory: Phonology, syntax, and acquisition (pp. 88-120). Oxford: Oxford University Press.

KATZ, S. M. (1987). Estimation of probabilities from sparse data for the language model component of a speech recognizer. IEEE Transactions on Acoustics, Speech, \& Signal Processing, 35, 400-401.

KELLER, F. (2001). Experimental evidence for constraint competition in gapping constructions. In G. Müller \& W. Sternefeld (Eds.), Competition in syntax (pp. 211-248). Berlin: Mouton de Gruyter.

Keller, F., \& AlEXopoulou, T. (2001). Phonology competes with syntax: Experimental evidence for the interaction of word order and accent placement in the realization of information structure. Cognition, 79, 301-372. 
Lapata, M., Keller, F., \& McDonald, S. (2001, July). Evaluating smoothing algorithms against plausibility judgements. In Proceedings of the 39th Annual Meeting of the Association for Computational Linguistics (pp. 354-361). Toulouse, France.

Lapata, M., McDonald, S., \& Kellek, F. (1999). Determinants of adjective-noun plausibility. In Proceedings of the 9th Conference of the European Chapter of the Association for Computational Linguistics (pp. 30-36). Bergen, Norway.

LEE, L. (1999). Measures of distributional similarity. In Proceedings of the 37th Annual Meeting of the Association for Computational Linguistics (pp. 25-32). College Park, MD.

LoRD, C. (1979). "Don't you fall me down": Children's generalizations regarding cause and transitivity. Papers \& Reports on Child Language Development, 17, 81-89.

Miller, G. A., Beckwith, R., Fellbaum, C., Gross, D., \& Miller, K. J. (1990). Introduction to WordNet: An on-line lexical database. International Journal of Lexicography, 3, 235-244.
ROBERTS, M. (2005). Lexical constraint learning. Unpublished doctoral dissertation, University of Warwick, Coventry, U.K.

ScHUTZE, C. T. (1996). The empirical base of linguistics: Grammaticality judgments and linguistic methodology. Chicago: University of Chicago Press.

SHEPARD, R. N. (1957). Stimulus and response generalization: A stochastic model relating generalization to distance in psychological space. Psychometrika, 22, 325-345.

SORACE, A. (2000). Gradients in auxiliary selection with intransitive verbs. Language, 76, 859-890.

SORACE, A., \& Keller, F. (2005). Gradience in linguistic data. Lingua, $115,1497-1524$.

TVERsKY, A. (1977). Features of similarity. Psychological Review, 84, 327-352.

(Manuscript received February 5, 2007; revision accepted for publication May 9, 2007.) 